\title{
La representación de personas LGTBI+ en prensa española conservadora y progresista
}

\author{
The Representation of LGTBI+ People by Spanish \\ Conservative and Progressive Press
}

\author{
Juan Ramón Guijarro-Ojeda (1) https://orcid.org/0000-0001-8453-2909 \\ Universidad de Granada, España,jrgo@ugr.es \\ Raúl Ruiz-Cecilia (D) https://orcid.org/0000-0002-8119-549X \\ Universidad de Granada, España, raulruiz@ugr.es
}

Recepción:

$16 / 06 / 2018$

Aprobación:

$10 / 01 / 2019$

\begin{abstract}
This paper investigates the way LGTBI+ people are represented by conservative and progressive Spanish newspapers. With this purpose in mind, a corpus has been compiled with news items published regarding the ratification of the legal nature of homosexual marriage in Spain on November 6, 2012. As a method, we have analyzed transitivity and the semantic roles of processes as an instrument of the Critical Discourse Analysis methodology. The main results reveal that there is a substantial difference between conservative and progressive newspapers: the prominence given by the conservative press to LGTBI+ people represents just one third of that displayed by the progressive press; the conservative press reduces the levels of consciousness, voice, cognition, desire, emotion and perception as compared to the progressive, which strengthens them. Finally, the conservative press uses negative processes as an LGTBI+ people's intrinsic feature; whilst the progressive uses them to reveal social injustice against them.
\end{abstract}

Key words: Critical Discourse Analysis, transitivity, LGTBI+, homosexual marriage, press.

Resumen: Este artículo investiga cómo las personas LGTBI+ son representadas por la prensa conservadora y progresista españolas en un corpus de noticias, en torno a la ratificación de la constitucionalidad del matrimonio homosexual en España el 06 de noviembre de 2012. La metodología de estudio es propia del Análisis Crítico del Discurso: roles semánticos de los procesos verbales, según el sistema de transitividad de la Lingüística Funcional. Los principales resultados revelan que existe una diferencia sustancial en el modo en que la prensa conservadora representa al colectivo LGTBI+ respecto de la progresista: la conservadora da significativamente menos protagonismo a estas personas en comparación con la progresista; la conservadora les reduce los niveles de conciencia, voz, cognición, deseo, emoción y percepción frente a la progresista que los potencia sustancialmente. Por último, la negatividad de los procesos es utilizada por la prensa progresista para denunciar injusticia social, y la prensa conservadora para potenciar lo negativo de este grupo.

Palabras clave: Análisis Crítico del Discurso (ACD), transitividad, LGTBI+, matrimonio homosexual, prensa. 


\section{Introducción}

Este trabajo analiza la forma en que dos periódicos españoles conservadores (abc.es, larazon.es) y otros dos progresistas (elpais.com, publico.es) representan a las personas $\mathrm{LGTBI}+{ }^{1}$, con motivo de la sentencia ratificatoria de la constitucionalidad del matrimonio homosexual del 6 de noviembre de 2012 por parte del Tribunal Constitucional (TC) (Boletín Oficial del Estado, 2012). La historia comienza en 2005 con la interposición de un recurso de inconstitucionalidad por parte de más de 50 diputados del grupo parlamentario Popular a la Ley 13/2005, de 1 de julio, por la que se modifica el Código Civil en materia de derecho a contraer matrimonio, aprobada por el gobierno del Partido Socialista Obrero Español (PSOE) presidido por José Luis Rodríguez Zapatero. En esta ley se podía leer: "El matrimonio tendrá los mismos requisitos y efectos cuando ambos contrayentes sean del mismo o de diferente sexo" (Artículo único, punto 1) (BOE, 2005).

Con el fin de conocer la manera como las personas LGTBI+ son representadas contrastivamente por la prensa conservadora y la progresista españolas, se llevará a cabo un análisis de la transitividad con foco en los roles semánticos de los procesos verbales, según los postulados de la Lingüística Funcional, cuya última edición la encontramos en Halliday y Matthiessen (2014), que articula Fairclough (2003) como instrumento adecuado para la metodología del Análisis Crítico del Discurso (ACD).

Nos interesa especialmente el estudio científico de los medios de comunicación por su rol como agentes de socialización que, junto a la familia o el sistema educativo, son esenciales en la sociedad de la información actual. Cuando realizan los reportajes de la sociedad, al mismo tiempo, están configurando esa realidad social desde su discurso. Los procesos de interiorización y exteriorización de la cultura y su amplio espectro de compartimentos encuentran en los medios una vía de singular poder (SánchezGarcía, 2012; Enguix, 2013).

Los teóricos del ACD como van Dijk (2009) se hacen eco de esta idea y reclaman la necesidad de un estudio científico sistemático de la prensa y sus modos de transmitir la información, porque influyen en la configuración de

1 La Federación Estatal de Lesbianas, Gays, Transexuales y Bisexuales (FELGTB) de España utiliza normalmente el acrónimo LGTB, si bien en numerosos de sus discursos y, dependiendo del agente, introducen la "I" para recoger también la Intersexualidad. Nosotros hemos incluido también el signo +, como empieza a generalizarse su uso por los colectivos más progresistas en reconocimiento aperturista de cualquier otro posicionamiento individual o colectivo de identidad sexual no contemplada en el acrónimo normalizado. 
lo que conocemos y creemos y cómo lo conocemos y creemos. En la actual "sociedad de la información", el seguimiento de noticias a través de medios escritos y audiovisuales corre paralelo en nivel de recursividad a las propias conversaciones con personas cara a cara. Esos contenidos informativos que circulan son discursos lingüísticos culturales y vehículos de los mismos a la vez y, por ende, existe mediatización por parte de los agentes productores y también por los receptores.

\section{Objetivos}

Con esta investigación se persiguen los siguientes objetivos: 1) Aplicar el estudio de la transitividad, recurso clave del Análisis Crítico del Discurso, como instrumento de representación de las personas LGTBI+ en el discurso periodístico; y 2) comparar prensa conservadora y progresista españolas en términos del empleo discursivo de la transitividad, para comprobar si existen diferencias entre ambas en el modo de representar a las personas LGTBI+.

\section{Fundamentación teórica}

\section{Contextualización social}

Se analizará ahora cómo se llega social y jurídicamente a la aprobación de la referida Ley 13/2005 en España por parte del gobierno progresista del PSOE, así como a la ratificación de su constitucionalidad el 6 de noviembre de 2012 por parte del Tribunal Constitucional. A todas luces ha sido el resultado de un largo proceso de reconfiguración social desde el fin de la dictadura franquista (1939-1975), que Calvo (2010) fija en tres pilares fundamentales:

[...] la culminación de una política de reconocimiento de derechos civiles que es consecuencia de las decisiones estratégicas del principal peticionario (el movimiento de gays y lesbianas), del ensanchamiento de la estructura de oportunidades (provocado por transformaciones tanto domésticas como en el nivel internacional) y de la existencia de una serie de condiciones que llevaron al PSOE a utilizar la oportunidad que las estrategias del movimiento social de lesbianas y gays y la evolución del contexto habían colaborado a generar (Calvo, 2010: 140).

En los años de democracia la sociedad española ha experimentado una apertura hacia la diversidad sexual realmente encomiable, pues durante los 40 años de dictadura y de Estado confesional la homosexualidad fue reprimida legal y policialmente, considerándose como peligro social y delito de 
escándalo público e, incluso, como un defecto físico o enfermedad. Así se regulaba en la Ley de Vagos y Maleantes (1933, reformada en 1954), en el Código de Justicia Militar (1945) o en la Ley de Peligrosidad y Rehabilitación Social (1970) (Martín, 2011).

En 1978 se establece democráticamente la Constitución española que posibilita el paso del Estado confesional al Estado democrático y laico. No obstante, aún en 1979, se suscribe el Acuerdo de Asuntos Jurídicos con la Santa Sede que supone un peso importante para perpetuar socialmente el matrimonio canónico, pero el progresivo desarrollo de la secularización en España permitirá finalmente romper con pilares matrimoniales como indisolubilidad-sexualidad-procreación como axis inescindible y dar paso a la dignidad, libre desarrollo y afectividad como bases del matrimonio. Igualmente, se va rompiendo con la visión tradicional de la homosexualidad como pecado (Fernández-Coronado, 2006).

El contexto internacional juega un papel esencial en España y fundamentalmente lo es su incursión de pleno derecho en la Unión Europea en 1986. Esta última resulta capital con regulaciones como la Resolución del Consejo de Europa, del 1 de octubre de 1981; Resolución del Parlamento Europeo, del 13 de marzo de 1984; Resolución del Parlamento Europeo, del 8 de febrero de 1994 o la Carta de los Derechos Fundamentales de la Unión Europea del año 2000. En todas ellas se promueve erradicar la discriminación de cualquier tipo del colectivo LGTBI+. En consecuencia, en varios países de la Unión Europea se aprueban leyes de parejas con total equiparación entre las homosexuales y las heterosexuales: Dinamarca 1989, Noruega 1993, Suecia 1994, Holanda 2001 o Bélgica 2003 (Calvo, 2010).

En España, en el plano político, cabe destacar la labor fundamental de Izquierda Unida (IU), que desde 1986 ha incluido sin falta en sus programas electorales la aprobación de este derecho. El PSOE no lo haría hasta las elecciones de 2000 y más específicamente en el programa de 2004:

El PSOE irá apuntando primero y, desarrollando después, un discurso y una estrategia basada en la necesidad de vincular el cambio ideológico con nuevas referencia basadas en el respeto a los derechos humanos, la extensión de los derechos civiles y la conquista de la ciudadanía plena (Calvo, 2010: 159).

Otro hecho importante en España acaece cuando el 28 de febrero de 1994 el alcalde de Vitoria (País Vasco) abre el primer registro de parejas de hecho, donde el sexo u orientación sexual no era relevante, sino el hecho de ser una unión estable. A esto se une una dura sentencia del Tribunal Supremo (STS 66/1994), del 28 de febrero, que denegaba la pensión de viudedad a parejas no casadas, lo cual causó un gran revuelo social y mediático 
(Boletín Oficial del Estado, 1994). Este caldo de cultivo, unido a la pérdida de mayoría absoluta parlamentaria, hace que el PSOE dé un giro hacia esta realidad y se ponga del lado de la comunidad LGTBI+, afianzando sus ideales de partido político progresista. También se apunta como hecho fundamental la creación de grupos de trabajo LGTBI+ dentro de los partidos políticos de ámbito nacional como en IU y el PSOE e, inclusive, en el Partido Popular (PP) conservador (Calvo, 2010).

Ahora se analizará la postura que adoptan los diferentes agentes sociales implicados en este proceso de cambio. En cuanto a los grupos sociales LGTBI+ y su movilización, se distinguen dos posicionamientos. En primer lugar, una gran parte del colectivo (COLEGAS, COGAM, Triángulo, Arcópolis, etc.) muestra su apoyo a la igualdad matrimonial, porque supone un avance de siglos, el fin de la homofobia legislativa, económica, etc. Aún así, reclaman que todavía queda mucho por luchar en el ámbito educativo o la plena aceptación social, y que el hecho de poder casarse también conllevaría el peligro de pensar que ya está todo conseguido socialmente (Etxazarra, 2007).

Otros grupos LGTBI+ como Gaytasuna (País Vasco) afirman que "el matrimonio es una estructura patriarcal, heterosexual... y nosotros entendíamos que tampoco teníamos que repetir esa..." (E.2) o que el "reconocimiento de la superioridad de la sociedad heterosexual que conlleva, inevitablemente, una inferioridad del hecho de ser gay o lesbiana. Desde esta perspectiva, no se está proponiendo transformar la sociedad, sino fusionarse en la ya existente" (D. 2) (Etxazarra, 2007: 11-12). Abogan más bien por las libertades individuales, manifestarse y expresarse como se quiera, y que la estructura del matrimonio no es la única a seguir. Asumir la institución del matrimonio representa para ellos un modelo de sociedad heteronormativa, aunque reconocen que a través de esa ley se puede acceder a una serie de derechos sociales que no se podría hacer de otra manera.

A este respecto, Tudela-Sancho (2017) conceptualiza la heteronormatividad como la configuración cultural del sistema sexo-género donde el modelo privilegiado socialmente es el heterosexual. Esto tiene consecuencias en los binomios masculino-femenino, instinto-voluntad, espacio público-espacio privado, naturaleza-sociedad, amor natural-amor mórbido, donde el hombre y lo masculino siempre primará sobre lo femenino: el varón es el gobernante familiar, el matrimonio es entre hombre y mujer, monógamo y fiel, y la familia cristiana y virtuosa.

Existe igualmente una fuerte agitación social en contra de la aprobación de la ley capitaneada por tres frentes: la Iglesia católica, el PP y el Foro 
Español de la Familia. ${ }^{2}$ El 18 de junio de 2005 celebraron una manifestación multitudinaria en Madrid en contra de la aprobación de la citada ley:

El PP se ocupaba de combatir la reforma en el campo político y legislativo, el Foro de la Familia se encargó de mantener vivo ese "no" a nivel social con movilizaciones y actos de protesta a pie de calle, mientras la Iglesia daba cobertura "moral" a esa oposición (Etxazarra, 2007: 18).

La postura de la Iglesia católica y del Foro de la Familia sobre lo que es una familia coincide en su componente heterosexual, procreativo y de consanguinidad (Extazarra, 2007: 16). El PP, por su parte, deja claro que no es una oposición a los homosexuales, sino al uso del término "matrimonio", pues lo entienden como hombre-mujer, según se especifica en la Constitución española de 1978. Buscan otro tipo de nomenclatura y regulación jurídica para las personas homosexuales. No obstante, también encontramos dentro del PP la Plataforma Popular Gay que es disidente en este sentido junto a otros poderosos políticos de primera fila como Esperanza Aguirre, Celia Villalobos o Alberto Ruiz Gallardón que ofició bodas homosexuales.

Con este panorama social sobre el terreno de juego, la ley es finalmente aprobada en el Congreso de los Diputados el 30 de junio de 2005 con 187 votos a favor, 147 en contra, 4 abstenciones y 12 ausencias de diputados. Tras esta situación, más de 50 diputados del PP interponen un recurso de inconstitucionalidad, alegando que las uniones entre personas del mismo sexo "no son matrimonio, ni por consiguiente puede configurarse jurídicamente como tal”. Siendo así, esta ley es contraria a la Constitución y su artículo 32.1 que establece: "El hombre y la mujer tienen derecho a contraer matrimonio con plena igualdad jurídica”.

No obstante, el artículo 32.2 hace constar que "la ley regulará las formas de matrimonio", hecho que ignora el PP en su recurso. Argumentaron también que la nueva fórmula desnaturalizaba el matrimonio o que la Real Academia Española (RAE) igualmente aludía a la unión de hombre y mujer en su definición de matrimonio exclusiva; hecho que cesó el 22 de junio de 2012 y que pasó a añadir también la acepción: "2. En determinadas legislaciones, unión de dos personas del mismo sexo, concertada mediante ciertos ritos o formalidades legales, para establecer y mantener una comunidad de vida e intereses". El PP siempre argumentó que la comunidad LGBTI+ podía recurrir a la legalidad de las "uniones civiles" y no necesariamente a la del matrimonio (García-Pedraz, 2012; Bonilla, 2013).

2 Fundado en 1999, representa a cuatro millones de familias que defienden los valores tradicionales de la persona y la familia, en el sentido cristiano-católico. 
Durante los siete años que discurren desde 2005 (aprobación de la ley) a 2012 (ratificación de constitucionalidad), aparecen estudios sociológicos relevantes que confirman la plena aceptación del matrimonio igualitario en la sociedad civil española. En 2007, la Fundación BBVA publica el Estudio Fundación BBVA sobre Actitudes Sociales de los Españoles (Fundación BBVA, 2007: 7-8). Por un lado, el 57,6\% acepta el matrimonio entre personas del mismo sexo; el 29,9\% lo rechaza y el 10,9\% muestra neutralidad; y, por otro, el $44 \%$ ve con buenos ojos que las parejas del mismo sexo puedan adoptar, frente al $42 \%$ que está en contra, restando un $14 \%$ que muestra neutralidad. La encuesta del Centro de Investigaciones Sociológicas (CIS) sobre las Actitudes de la juventud ante la diversidad sexual (CIS, 2010) concluye que el 76,8\% de los jóvenes valoran el matrimonio homosexual de forma positiva.

En cuanto a la cobertura ofrecida por la prensa española sobre el matrimonio homosexual, Guijarro-Ojeda (2015) confirma que los periódicos cumplen con sus líneas editoriales y que los conservadores desplegarán un amplio abanico de recursos discursivos contrarios al matrimonio homosexual y los progresistas a favor. Se destaca la voz que la prensa conservadora da al PP y a la Iglesia; mientras que la prensa progresista expone principalmente las voces del colectivo LGTBI+, seguido de los miembros del PP partidarios de la ley. También analiza los temas tratados en estos periódicos y los clasifica en: favorables a la ley (matrimonio homosexual, sentencia del TC, personas LGTBI+, ideología de género o PSOE) y contrarios a la ley (PP, Iglesia, ultraderecha o el recurso del PP).

Los resultados cuantitativos confirman las líneas editoriales y revelan que los elementos pro-LGTBI+ son evaluados positivamente por los conservadores en un $24,6 \%$ de los casos, mientras que los progresistas lo hacen en un $75,4 \%$, o sea el triple de casos. Si nos fijamos en los elementos contra-LGTBI+ y en su valoración positiva, los conservadores lo hacen en un $45,8 \%$ de los casos y los progresistas con un mínimo 5,1\%.

Finalmente, el Tribunal Constitucional falló de manera favorable sobre la constitucionalidad del matrimonio homosexual el 6 de noviembre de 2012, que en aquel momento estaba compuesto por siete magistrados de orientación progresista y cinco de orientación conservadora. Contó con ocho votos a favor ( 7 progresistas +1 conservador) y tres votos en contra ( 3 conservadores) con sus correspondientes votos particulares. El magistrado conservador Francisco Hernando se abstuvo de participar en el proceso, porque en su etapa anterior como presidente del Consejo General del Poder Judicial (CGPJ) había tomado decisiones contrarias a la ley. 
La sentencia del TC, en palabras de Bonilla (2013: 41), recoge fundamentos sociales que permean lo jurídico de la Constitución de 1978 en una interpretación evolutiva de la sociedad, porque en aquel momento no había la demanda social que sí existe en 2005:

Más de 30 años después, el TC interpreta que la imagen actual del matrimonio en España, producto de la evolución social, se define como una comunidad de afecto o sociedad de ayuda mutua que genera un vínculo entre dos personas, que poseen idéntica posición en el seno de esa institución, y que voluntariamente, deciden unirse en un proyecto de vida familiar común, prestando su consentimiento respecto de los derechos y deberes que conforman la institución y manifestándolo expresamente mediante las formalidades establecidas en el ordenamiento (Bonilla, 2013: 41).

En los votos particulares de los tres magistrados conservadores se esgrimen argumentos variados y comunes, como que, entre otras, la función del matrimonio es la perpetuación de la especie y ahora queda desnaturalizado. La nueva ley es contraria también a los intereses de los menores que puedan ser adoptados por las parejas homosexuales. Así, contravendrían la configuración constitucional de la filiación y atentaría contra el interés del menor, porque existe relación afectiva y sexual entre los padres o madres que es vida privada pero no vida familiar. No se acepta el cambio conceptual del matrimonio como figura socialmente relevante a otra individualista de emancipación inconexa con la sociedad. Tampoco aprueban que sean las conductas sociales las que condicionen la interpretación constitucional y estiman que las relaciones homosexuales deberían ser reguladas al margen de la institución matrimonial (BOE, 2012: 202-219).

\section{Análisis Critico del Discurso y transitividad}

El objetivo principal del ACD consiste en analizar el discursivo en sus niveles textual y estructural, para descifrar cómo esta arquitectura lingüística produce componentes culturales como la manipulación, la ideología, el poder, la autoridad o la desigualdad. Resulta pertinente aquí la definición que van Dijk (2009) articula sobre lo que entiende por ACD:

[...] un tipo de investigación analítica sobre el discurso que estudia primariamente el modo en que el abuso del poder social, el dominio y la desigualdad son practicados, reproducidos, y ocasionalmente combatidos, por los textos y el habla en el contexto social y político. El Análisis Crítico del Discurso, con tan peculiar investigación, toma explícitamente partido y espera contribuir de manera efectiva a la resistencia contra la desigualdad social (van Dijk, 2009: 149). 
Para el ACD que aquí presentamos centrado en el estudio de la transitividad nos valemos de la Lingüística Funcional, que establece tres metafunciones del lenguaje según Halliday y Matthiessen (2014): ideativa, interpersonal y textual. Nos interesa la función ideativa que establece que la oración es una representación. A través de ella, las personas representan su experiencia interna y externa del mundo real (función experimental); así como también permite el establecimiento de relaciones lógicas (función lógica). Esta función se realiza en la oración mediante el sistema de transitividad, donde se expresan los procesos (verbos), los roles de los participantes y las circunstancias que los condicionan. Cada uno, en la elección que hace de estos elementos a la hora de representar la realidad, se posiciona ideológicamente en la proyección de su punto de vista sobre esa realidad (Fairclough y Fairclough, 2012).

Se establecen seis tipos de procesos que reflejan modos diferentes de experiencia y conllevan distintos tipos de participantes: 1) material: hace referencia a acciones y sucesos concretos y abstractos e implica la idea de cambio. Los participantes son actores, metas, receptores y clientes; 2) mental: se relaciona con la experiencia interna de una persona con el mundo que le rodea e implica nociones de emoción, cognición, percepción y deseo. El participante puede ser: sensor (alto grado de conciencia) o fenómeno (cualquier participante, consciente o no, oído, gustado, comprendido, etc.); 3) relacional: implica la experiencia externa e interna y las nociones de ser y/o llegar a ser. El participante puede ser: transportador, atributo, benefactor o el que atribuye; 4) verbal: se refiere a los intercambios simbólicos de significados. Los participantes son "dicentes", personas que en potencia actúan verbalmente sobre otras personas, ideas o cosas. También podríamos encontrar los roles participantes de receptor, dicho (verbiage) y objeto; 5) conductual: representan un comportamiento psicológico o fisiológico como ver o reír. El participante primordial es "el que se comporta"; y 6) existencial: implica la existencia de $\mathrm{X}$, conocido como 'existente' y puede ser una persona, institución, evento, objeto, abstracción o acción (Halliday y Matthiessen, 2014: 247-307).

\section{Estudios previos}

Aunque la literatura científica existente en torno a la representación de las personas LGTBI+ en la prensa no es demasiado prolija, sí nos ofrece un abanico de perspectivas temáticas y metodológicas que constatan cómo la discriminación explícita y latente está presente en los discursos culturalesmediáticos. 
En su estudio sobre la visibilidad de las relaciones homosexuales en el contexto europeo de Italia, Francia y España, Carratalá (2011) apunta que goza cada vez de más importancia en la esfera pública y en los medios de masas. Sin embargo, revela que aún hay un abanico amplio de rasgos de homofobia velada para referirse a las parejas homosexuales, que aflora en forma de eufemismos que reproducen rasgos estigmáticos. Estos hallazgos vienen a demostrar la presencia de un heterocentrismo dominante y hegemónico, que sigue desplegando una centralidad discursiva privilegiada heterosexual.

En el mismo sentido, Clarke (2006) demuestra que en Canadá existe un sesgo latente en la intención de la prensa en representar al colectivo LGTBI+ asociado a la epidemia del VIH, ignorándose factores tan relevantes como la pobreza, el racismo o la prevención. En esta línea, Enguix (2013) analiza la mediación mediática y el activismo LGTB a través del estudio del impacto en prensa escrita y medios sociales de la celebración del orgullo en las ciudades de Madrid y Barcelona en 2010. Concluye que la prensa utiliza estrategias de descontextualización y estereotipación, siendo partícipe de una construcción sociocultural de alteridad y hegemonías sociales. Cabe destacar el dato de que lo LGTB gira hegemónicamente en torno a lo "gay" (hombres) y lesbianas, bisexuales o transexuales permanecen mayoritariamente silenciados (Enguix, 2013: 43).

Un estudio central paralelo viene de manos de Jowett y Peel (2010), quienes realizan un análisis del Civil Partnership Act, 2004 desde una perspectiva socioconstructivista, para ver cómo lo cubre la prensa escrita británica. Con tal fin, se examinan durante tres meses (noviembre 2005-junio 2006) 348 periódicos considerando las siguientes variables: estatus legal de las parejas homosexuales respecto de las heterosexuales; la centralidad del factor "heterosexualidad" en el matrimonio y la legalización del matrimonio entre personas del mismo sexo por el Civil Partnership Act. Los hallazgos son contundentes y anuncian que la heteronormatividad es la norma en la representación de esta temática y no deja espacio para reconocer y construir relaciones más radicales entre personas del mismo sexo.

En relación con la forma en que el Chicago Tribune y The New York Times, conservador y progresista, respectivamente, cubren la temática de los matrimonios homosexuales en Estados Unidos, Pan et al. (2010) se centran en el estudio del framing de las informaciones sobre el matrimonio entre personas del mismo sexo, con base en la ideología de cada cabecera periodística. El Chicago Tribune focaliza en el tema de la moralidad y su incidencia en el debate social donde el veredicto favorable de los tribunales no hace reorientar su discurso y continúa con la defensa de los valores americanos de la 
tradición y la familia. Por su parte, The New York Times centra su interés en promocionar los valores de igualdad y defiende activamente el matrimonio homosexual después de que el estado de Massachusetts lo legitimara.

Por su parte, Strand (2012) estudia los periódicos locales Daily Monitor (privado) y New Vision (público) tras la aprobación del Anti-Homosexuality Bill en Uganda en 2009, con el objeto de analizar la construcción discursiva homofóbica de las personas LGTBI+. Ambas cabeceras, siendo de orientaciones editoriales divergentes, reproducen la homofobia arraigada en ese contexto cultural; si bien es cierto que el Daily Monitor avanza críticas contra este decreto discriminatorio e intenta deslegitimizar la institucionalización de la homofobia y el impacto peyorativo que ejerce sobre los ciudadanos y lectores.

Gouveia (2005) disecciona la forma en que el Diário de Notícias, prensa seria portuguesa, representa a gays y lesbianas. Entre otros recursos lingüísticos sistémico-funcionales, utiliza la transitividad para estudiar la forma en que los homosexuales son representados en un país donde tienen poca visibilidad y poder. Concluye con el resultado de que los gays son representados mayoritariamente, en un $65 \%$ de los casos, en procesos relacionales atributivos, lo cual los confina a un espacio claramente de inacción. Siendo así, los gays no son representados como entidades concretas realizando actividades concretas, sino que les son otorgados ciertos atributos como cualidad, circunstancia o posesión. Muy de lejos, en un 20\% de los casos, son las metas de procesos materiales, y en un 15\% de los casos, en procesos existenciales. Sorprendentemente, no identifica casos de homosexuales participantes en procesos mentales, verbales o conductuales, lo cual viene a confirmar una clara velación de este colectivo en el discurso público.

El trabajo de Bartley e Hidalgo-Tenorio (2015) estudia la construcción discursiva de la homosexualidad en la prensa durante el Celtic Period en Irlanda a través de la transitividad. Los resultados revelan actitudes negativas por parte de la Iglesia, donde abundan los procesos mentales negativos emotivos en los que los gays son fenómenos o los procesos verbales donde los temas relacionados con la homosexualidad son representados como verbiage o "dichos" con implicaciones negativas. De igual manera, por parte de políticos Unionistas Democráticos, los procesos relacionales son de naturaleza evaluativa negativa. Otro aspecto revelado de especial calado consiste en conectar actos homofóbicos con actividades criminales y no claramente con un ataque a la orientación sexual, como en otros países del entorno. Esta cuestión supone el velado religioso de una realidad de marginalización y represión discursiva. 
En línea con la represión religiosa, Acevedo y Elgueta (2008) llegan a la conclusión de que, en contra de lo que marcaría la lógica, la prensa de izquierdas en Chile es transmisora de discursos homofóbicos por apoyo a la Unidad Popular. Justifican esta cuestión por la influencia masiva de la religión católica en América Latina que es un eje transversal tanto para la prensa conservadora, como para la progresista. También, por su parte, Peterson (2011) muestra cómo una organización cristiana en Estados Unidos incide en la amenaza que suponen los homosexuales y sus formaciones-relaciones de parentesco para el capital humano, por basarse estas en relaciones de afectividad contrarias a los preceptos socioeconómicos del neoliberalismo y su modelo de familia ideal.

\section{Metodología}

\section{Corpus de estudio}

El corpus de estudio total está conformado por 55 elementos informativos de cuatro diarios españoles de tirada nacional en sus versiones digitales. Su orientación ideológica es: prensa conservadora (abc.es y larazon.es) y prensa progresista (elpais.es y publico.es). La distribución por número de piezas informativas es como sigue: 17 corresponden a abc.es; 13 a larazon.es; 14 a elpais.es y 11 a publico.es. ${ }^{3}$ Si consideramos el número de palabras, el corpus total asciende a 36.978 , que se distribuyen en 18.501 (cabeceras conservadoras) y 18.477 (cabeceras progresistas). El gran cuerpo de las informaciones seleccionadas datan desde el 8 de febrero de 2012 hasta el 23 de abril de 2013, con la salvedad de una sola noticia fechada el 21 de septiembre de 2005 . La agitación del debate es relativamente importante en los periodos que preceden y suceden al día del fallo del Tribunal Constitucional.

Se han compilado aquellas piezas informativas relacionadas con el tema de estudio, resultado de la introducción en los buscadores en línea de estos periódicos de los siguientes términos de búsqueda: matrimonio entre personas del mismo sexo; matrimonio homosexual; matrimonio gay y matrimonio igualitario. Introdujimos diferentes variables del mismo término para abarcar todo el espectro posible en torno a esta cuestión. Una vez cotejados todos los resultados de los diferentes términos de búsqueda, pudimos crear el corpus final integrado por los géneros periodísticos de editoriales, columnas de opinión, noticias y comentarios de personalidades invitadas o de periodistas.

3 Los datos ilustradores del corpus en la sección 5. Resultados y análisis serán identificados con el nombre del periódico, seguido del número que corresponde a la pieza informativa dentro de cada cabecera, tal y como se indica aquí. 


\section{Variables de estudio}

Siguiendo las indicaciones metodológicas de Richardson (2007), fundamentado en la corriente social del ACD, las variables de estudio que se fijan para el análisis del corpus son: 1) Tipología, frecuencia y valor (positivo-neutro y negativo) de los procesos donde intervienen directamente personas LGT$\mathrm{BI}+$, bien en roles agentes o pacientes de los procesos materiales, mentales, relacionales, verbales, conductuales y existenciales; y 2) Frecuencia y nivel de identificación de los participantes en los procesos.

\section{Método}

Una vez compiladas las piezas informativas, se procedió a la identificación de los procesos de todo el corpus de manera individual e independiente por parte de los dos investigadores. Con este fin en mente, se han considerado aquellos procesos verbales cuyos participantes son personas LGTBI+ o grupos/ asociaciones de las mismas. Una vez que cada investigador tuvo su parrilla de resultados lista, se cotejaron ambas para ver los puntos de discrepancia y hallar un punto de convergencia final.

\section{Resultados y análisis}

En primer lugar, presentamos los resultados parciales de los subcorpus conservador y progresista, para dar finalmente paso a la comparativa entre ambos.

\section{Subcorpus conservador}

La Tabla $1^{4}$ presenta la tipología y número total y porcentaje de procesos correspondientes al corpus conservador. En él se han registrado un total de 80 procesos donde intervienen directamente personas LGTBI+. El tipo de proceso identificado como mayoritario corresponde al material y cubre un $71,25 \%$ (82,5\% positivo-neutro y $12,5 \%$ negativo) del total del corpus.

- Personas LGTBI+ [actores] han rodeado [proceso material] la estatua del oso y el madroño con una bandera gigante del arco iris para conmemorar el amparo del "matrimonio igualitario" [valor positivo-neutro] (abc.es, 13)

\footnotetext{
4 Las tablas y el gráfico se encuentran en el Anexo, al final del presente artículo (N. del E.).
} 
- [...] más de 20.000 parejas homosexuales [actores] han contraido matrimonio [proceso material] [valor positivo-neutro] (larazon.es, 1)

- "No le cedo elpaso [proceso material] a un maricón [meta]" (larazon. es, 6)

- $\quad[. .$.$] homos y lésbicas [actores] hicieron [proceso material] de su sexo$ un sayo sin que sus sociedades se alarmaran [valor negativo] (larazon. es, 6)

Le siguen con igualdad de porcentajes los procesos mentales con un $10 \%$ (62,5\% positivo-neutro y $37,5 \%$ negativo) y relacionales con un $10 \%(50 \%$ positivo-neutro y $50 \%$ negativo).

- La presidenta de una de las convocantes, la Federación Estatal de Lesbianas, Gais, Transexuales y Bisexuales (FELGTB), Boti García Rodrigo [sensor], ha alabado [proceso mental] la decisión del pleno del Constitucional [...] [valor positivo-neutro] (abc.es, 13)

- $\quad[. .$.$] en los medios audiovisuales sólo se hacen [proceso relacional] ricos$ y famosos los de la otra acera antropológica [benefactores] [valor negativo] (larazon.es, 6)

Por último, encontramos los procesos verbales con un 8,75\% (100\% positivo-neutro).

- Rafael Moral [dicente], felicitó [proceso verbal] a De la Torre "por su valentía política y empatía [...]" [valor positivo] (abc.es, 5)

- $[. .$.$] algunos de los gritos que corean [proceso verbal] los concentrados$ [dicentes] [gays y lesbianas] [valor positivo] (abc.es, 13)

No se han identificado procesos conductuales o existenciales. Si atendemos al valor positivo-neutro o negativo de los diferentes procesos, el cómputo general nos indica que el $65,35 \%$ de los procesos son de carácter positivo o neutro, frente al $21,25 \%$ que son de carácter negativo.

Entre los participantes que realizan o reciben la acción de los procesos en el corpus conservador se puede apreciar un amplio espectro de personas o colectivos LGTBI+. Algunos de estos participantes son personas célebres por sus trabajos como Ricky Martin, Johanna Sigurdaddottir (primera ministra islandesa), Elton John, el emperador romano Julio César, Boti García Rodrigo (activista LGTBI+ española), Phyllis Lyon y Del Martin (pareja conocida de lesbianas) o Rafael Moral (presidente de Colegas en la región de Andalucía, España). El resto de agentes LGTBI+ obedecen siempre a personas genéricas que se nombran de las siguientes maneras: parejas del mismo sexo, parejas gays y lesbianas, homos y lésbicas, los de la otra acera antropológica, pareja homosexual, dos hombres y dos mujeres, asociaciones/ colectivos LGTBI+, parejas, la homosexualidad, grupos de presión, gays y 
lesbianas, madre lesbiana, grupo de presión minoritario, dos personas del mismo sexo, el arcoíris, parejas de hecho o jóvenes con tendencias gays.

Si atendemos a la estadística, podemos cuantificar que de los 80 participantes implicados en los procesos del corpus conservador, los agentes identificados o conocidos representan el 20\% del total, mientras que los no-identificados o genéricos representan el $80 \%$ del total.

\section{Subcorpus progresista}

Por otra parte, la Tabla 2 recoge los procesos verbales que se han identificado en el subcorpus progresista. Como podemos apreciar, el número total suma 242 procesos. Por orden de frecuencia encontramos los procesos materiales con un $35,4 \%$ ( $79,1 \%$ positivo-neutro y $20,9 \%$ negativo):

- Los homosexuales [actores] se movilizan [proceso material] en defensa de sus familias [valor positivo-neutro] (elpais.es, 1)

- Los colectivos de gais [actores] celebran [proceso material] la inclusión del matrimonio homosexual en el DRAE [valor positivo] (elpais.es, 2)

- 'Por el matrimonio natural y cristiano, y a los maricones [metas] que les den [proceso material] por el ano' [valor negativo] (publico.es, 2)

Los procesos verbales representan un $29 \%$ (92,8\% positivo-neutro y 7,2 negativo):

- Esther [dicente] explica [proceso verbal] que su madre, [...], ahora presenta a su hija y su mujer con naturalidad [valor positivo] (elpais.es, 7)

- “ “Aquí solo puede estar el padre!”, le espetó [proceso verbal] el médico a Herminia [mujer lesbiana, receptora] [valor negativo] (elpais.es, 7)

Los procesos mentales, con un $24,4 \%$ (74,6\% positivo-neutro y $25,4 \%$ negativo), ocupan la tercera posición:

- Los colectivos de lesbianas, gays, transexuales y bisexuales [sensores] consideran [proceso mental] positiva e importante la decisión de la Real Academia de la Lengua (RAE) de incluir la unión de personas del mismo sexo en la definición de matrimonio [valor positivoneutro] (elpais.es, 2)

- Muchas familias [sensores] han vivido [proceso mental] con la angustia de saber si las iban a anular de golpe y porrazo [valor negativo] (elpais.es, 7)

- [Carlos y Emilio] [sensores] se enamoraron [proceso mental] bajo la amenaza de una ley de vagos y maleantes [...] [valor negativo] (elpais.es, 7) 
Los procesos relacionales se alzan con un $7,8 \%$ (68,4\% positivo-neutro y $31,6 \%$ negativo):

- "Si eres [proceso relacional] soltera o tu pareja [existente 1] es [proceso relacional] una mujer [existente 2] [...] [valor neutro] (elpais. es, 7)

- [...] los gays [existentes 1] son [proceso relacional] 'bijos [existentes 2] de padres hostiles, alcohólicos y distantes' y de 'madres sobreprotectoras' [...] [valor negativo] (publico.es, 2)

Por último, sin prácticamente representación, encontramos los procesos existenciales con un $2 \%$ ( $40 \%$ positivo-neutro y $60 \%$ negativo) y conductuales con tan solo $1,4 \%$ (100\% positivo-neutro).

- Antes de 2005, no éramos [proceso existencial] nadie legalmente" [Esther, mujer lesbiana] [existentes] [valor negativo] (elpais.es, 7)

- Durante estos siete años [Emilio Menéndez y Carlos Baturín, pareja gay] [los que se comportan] han experimentado [proceso conductual] la felicidad de un amor reconocido por la ley [...] [valor positivoneutro] (elpais.es, 7)

El 80,6\% de los procesos son catalogados de carácter positivo o neutro, frente al $19,4 \%$ de cualidad negativa.

En el corpus progresista, los participantes en las acciones de los diferentes procesos pertenecen tanto a personas con nombre propio, populares o no, como a referencias genéricas a personas LGTBI+. Entre los nombres propios, destacamos: Antonio Poveda y (presidente de FELGTB), Carla Antonelli (diputada PSOE y activista transexual), Pedro Zerolo (diputado del PSOE y activista LGTBI+), Rafael Salazar (presidente de COLEGAS), Boti García Rodrigo (conocida activista LGTBI+ española), José María Núñez (presidente de la Fundación Triángulo), Rubén López (coordinador del grupo gay universitario Arcópoli), María José Ariza (vicepresidenta de FLG) o Agustín López (portavoz de COGAM).

Cuando se hace referencia al colectivo LGTBI+ de manera genérica se emplean los siguientes términos: homosexuales, entidades/entidades LGTBI+, colectivos de gais, gays y lesbianas, asociaciones de gays y lesbianas, colectivos de gays y lesbianas, organizaciones, colectivos de lesbianas, gays, transexuales y bisexuales, parejas, marcha del orgullo, parejas homosexuales, personas gays, ciudadanos gays, asistentes (gays), quienes han contraído matrimonio (gay), familias con padres del mismo sexo, personas del mismo sexo, uniones entre homosexuales, familias gays, parejas del mismo sexo, maricones, gays, hijos de gays, uniones homosexuales o adultos (gays). 
De los 242 procesos identificados en el corpus progresista, el 65,7\% pertenece a participantes identificados o famosos, mientras que $34,3 \%$ son clasificables como no-identificados o genéricos.

\section{Análisis comparativo prensa conservadora-progresista}

En el Gráfico 1 se puede observar la comparativa de la representación de las personas LGTBI+ en ambos tipos de cabeceras, donde se evidencia una serie de diferencias que permiten apreciar con cierta claridad el modo como la prensa conservadora y la progresista representan a este colectivo. En dos corpus con casi idéntico número de palabras, llama la atención el número de procesos en los cuales intervienen personas LGTBI+ en la prensa conservadora y la progresista. En la primera se han identificado 80 procesos, mientras que en la segunda son 242. Es evidente que en la prensa progresista existe un interés triple por darles protagonismo, independientemente de los procesos a los que estén asociados.

Otro aspecto significativo reside en la distribución de frecuencias entre los diferentes procesos. En lo referente a los procesos materiales, en la prensa conservadora copan el $71,25 \%$ de los mismos, mientras que en la prensa progresista tan solo la mitad con un 35,4\%. Los procesos materiales implican conciencia o no por parte de los actores o metas y se refieren a acciones que producen cambios. En este sentido, queda patente que la prensa conservadora representa al colectivo LGTBI+ principalmente como personas que hacen cosas, con un nivel de conciencia de lucha contra la desigualdad bajo. Esto aparca los procesos mentales o verbales donde el grado de conciencia y afectividad de los participantes es muy elevado.

Una de las diferencias más significativas viene de la mano de los procesos mentales que implican la experiencia interna del mundo y nociones de cognición, deseo, emoción y percepción. Si se trata de personas LGTBI+, los datos son realmente reveladores, pues en el corpus progresista representan el 24,4\% de los procesos; mientras que en el corpus conservador tan solo el 10\%. Para la prensa progresista es importante destacar la humanización de este colectivo; en tanto que la conservadora prefiere no dotar de este carácter consciente y emocional al colectivo.

- A Carlos [sensor] le preocupa [proceso mental] que los jueces del Tribunal Constitucional sean propuestos por políticos y manifiesten su filiación ideológica (elpais.es, 7)

Estos procesos mentales son muy significativos en el corpus progresista, porque normalmente se utilizan para expresar la manera interna en que las 
personas LGTBI+ reaccionan ante la injusticia social de no ser reconocidos en igualdad de derechos que las heterosexuales.

En la misma línea del punto anterior, encontramos el caso de los procesos verbales. Mientras que en la prensa progresista ascienden al 29\% de los procesos, en la conservadora solo representan un $8,75 \%$.

- "Nos ponían pegas en el Registro, pensábamos que no nos podríamos casar", explica [proceso verbal] Herminia [dicente, lesbiana] (elpais.es, 7)

- Ricky Martin [dicente] dice [proceso verbal] que "si tocaran la ley del matrimonio gay $[\ldots]$ (abc.es, 3)

En este caso de lucha por derechos sociales, es muy relevante que la prensa progresista represente a las personas LGTBI+ como dicentes y les dé voz en la lucha por la igualdad, bien a título personal, como pareja o como colectivo. Por parte de la prensa conservadora, podríamos hablar de silenciamiento en línea con lo defendido por Carratalá (2010). Diferentes estudios dentro de la corriente de la lingüística queer (Motschenbacher y Stegu, 2013; Giffney y O'Rourke, 2017) inciden en que esta actitud de silenciamiento de las personas o eventos LGTBI+ es una de las principales estrategias discursivas de discriminación contra este colectivo.

$\mathrm{Si}$ atendemos al valor positivo-neutro y negativo de los procesos en ambos corpus, apreciamos que no hay diferencias realmente significativas en la frecuencia. La prensa conservadora otorga valor positivo-neutro al 78,75\% de los procesos, y la progresista a $80,6 \%$; en el lado negativo, la conservadora alcanza el valor de $21,25 \%$, y la progresista el $19,4 \%$. Ahora bien, existe una diferencia relevante sobre la intención de cada grupo de cabeceras en los valores negativos. La prensa conservadora representa a los LGTBI+ en papeles peyorativos, agentes o pacientes, y lo negativo en la prensa progresista tiene la intención de denuncia de acciones negativas hacia este colectivo:

- $\quad[. .$.$] hay solventes estudios que desaconsejan la adopción por parejas$ homosexuales, etc. (larazon.es, 12) [valor negativo]

Incluso en el caso: [...] unos "lobbies" que te apoyen [...] (larazon.es, 12), que en principio tiene un valor positivo, no es más que un juego discursivo donde predomina la ironía, y la intención es claramente negativa para cuestionar la acción de grupos de presión LGTBI+.

Como se ve en el siguiente ejemplo, en el caso de la prensa progresista se utiliza el factor negativo para denunciar la posición homofóbica del ex ministro del Interior, Fernández Díaz: [...] estoy en contra del derecho a adoptar niños por parejas del mismo sexo (elpais.es, 10) [valor negativo]. O en los casos de: "Los maricones que adopten ratones" (publico.es, 2) o "[...] la 
homosexualidad es un trastorno de la personalidad" (publico.es, 2), donde se reportan en estilo directo las palabras de personas homofóbicas en manifestaciones contra el matrimonio homosexual.

Por último, resulta muy reveladora la comparativa entre el porcentaje del nivel de identificación de los participantes en los diferentes procesos en los corpus conservador y progresista. Los participantes que se pueden identificar con nombre y apellidos o por asociaciones LGTBI+ normalizadas represen$\tan 20 \%$ en el corpus conservador, frente a $65,7 \%$ en el corpus progresista. En contrapartida, los participantes genéricos o que no pueden identificarse ascienden a la cifra de $80 \%$ en el corpus conservador, mientras que en el progresista tan solo copa un 34,3\%.

Estos datos tan dispares entre los dos subcorpus analizados vienen a confirmar los datos de los procesos analizados en este trabajo y que pueden interpretarse como un intento evidente por parte del corpus conservador de invisibilizar al colectivo LGTBI+; frente al corpus progresista que apuesta claramente por una visibilización de los participantes LGTBI+ con nombre y apellidos en torno al asunto capital de la constitucionalidad del matrimonio entre personas del mismo sexo en España.

El análisis de los resultados de este estudio confirma los resultados de investigaciones en los ámbitos nacional (Carratalá, 2010) e internacional (Gouveia, 2005; Jowett y Peel, 2010; Pan et al., 2010; Bartley e Hidalgo, 2015) sobre el tratamiento discursivo de las personas LGTBI+ o de la homosexualidad en sí misma. Por lo general, se constata la condición de represión discursiva en torno a este colectivo y sus derechos o, en el mejor de los casos, el despliegue de recursos eufemísticos veladores. Cuando se ha analizado contrastivamente prensa progresista y conservadora, como en el caso de Pan et al. (2010), nuestro estudio corrobora el patrón de que la prensa progresista defiende los valores de igualdad de derechos y, por ende, de defensa activa del matrimonio homosexual; en tanto que la prensa conservadora si bien no despliega un ataque frontal homofóbico, porque se podría tratar de ilegalidad en España, sí utiliza recursos discursivos para velar y despojar de sus derechos a este colectivo social.

\section{Conclusiones}

Si fijamos las conclusiones de este trabajo con base en los objetivos planteados al inicio del mismo, podemos afirmar que la aplicación de la transitividad como metodología instrumental para el $\mathrm{ACD}$ ha demostrado ser válida para desvelar los vericuetos discursivos de la prensa y, en este caso, entender cómo 
se representa mediática y socialmente a un colectivo de personas. El recurso lingüístico y discursivo de la transitividad ha sido utilizado consciente o inconscientemente por ambos tipos de cabeceras, y lo cierto es que los resultados hallados coinciden con lo que socialmente se espera de cada línea editorial.

El estudio de los diferentes procesos que despliega cada corpus ha revelado datos significativos sobre cómo ambos tipos de cabeceras representan al colectivo LGTBI+. La prensa conservadora da poco protagonismo a estas personas en su discurso público; las desprovee de cierto tipo de acción consciente y, al mismo tiempo, las dota mínimamente de cualidades humanas como la cognición, la percepción, el deseo o la emoción. Igualmente, les niega la voz en los planos individual, de pareja o como grupo, y el carácter negativo de los procesos donde son implicados es presentado como intrínseco de este colectivo.

Por otro lado, la prensa progresista da un enorme protagonismo a las personas LGTBI+, las dota de conciencia y voz, y de cualidades humanas de cognición, deseo, emoción y percepción. La intención del uso de negatividad en los procesos obedece claramente a un propósito de denuncia social de actitudes homofóbicas o heteronormativas hacia este grupo de personas.

Con todo esto, queda patente la profundidad cultural de actitudes negativas como la homofobia o la heteronormatividad. En el caso español, han pasado ya 40 años desde que se celebrara la primera manifestación de lucha por la liberación de la homosexualidad en 1977 (Enguix, 2017), y en nuestros días se siguen reproduciendo discursos negativos de forma manifiesta o latente, como los que han visto la luz a través de este estudio. Todo esto en un país donde en 2014 en la celebración del Orgullo LGTBI+ en Madrid se dieron cita, según los organizadores, 1,2 millones de personas.

\section{Referencias}

Acevedo, Claudio y Elgueta, Eduardo (2008), "El discurso homofóbico en la prensa izquierdista durante la Unidad Popular", en Revista Izquierdas, núm. 3, Chile: Instituto de Estudios Avanzados de la Universidad de Santiago de Chile.

Bartley, Leanne e Hidalgo-Tenorio, Encarnación (2015), "Constructing perceptions of sexual orientation: A corpus-based Critical Discourse Analysis of transitivity in the Irish Press", en Estudios Irlandeses, núm. 10, España: Universidad de Almería.

Boletín Oficial del Estado (1994), Sentencia 66/1994, de 28 de febrero de 1994, recurso de amparo 1.714/1992, contra Sentencia de la Sala de lo Social del Tribunal Supremo, recaída en autos de pensión de viudedad, España: Gobierno de España. Disponible en: https://www.boe.es/boe/dias/1994/03/24/pdfs/T00044-00047.pdf [10 de marzo de 2018]. 
Boletín Oficial del Estado (2005), Ley 13/2005, de 1 de julio, por la que se modifica el Código Civil en materia de derecho a contraer matrimonio, España: Gobierno de España. Disponible en: https://www.boe.es/boe/dias/2005/07/02/pdfs/A23632-23634.pdf [10 de marzo de 2018].

Boletín Oficial del Estado (2012), Sentencia 198/2012, de 6 de noviembre de 2012. Recurso de inconstitucionalidad 6864-2005, España: Gobierno de España. Disponible en: https://www.boe.es/boe/dias/2012/11/28/pdfs/BOE-A-2012-14602.pdf $\left[\begin{array}{ll}15 & \text { de }\end{array}\right.$ marzo de 2018].

Bonilla, Juan José (2013), “Sobre la declaración de constitucionalidad del matrimonio entre personas del mismo sexo, homosexual o igualitario", en Espacio y Tiempo, Revista de Ciencias Humanas, núm. 27, España: Universidad de Sevilla.

Calvo, Kerman (2010), "Movimientos sociales y reconocimiento de derechos civiles: la legalización del matrimonio entre personas del mismo sexo en España”, en Revista de Estudios Politicos, núm. 147, España: Centro de Estudios Políticos y Constitucionales.

Carratalá, Adolfo (2011), "La representación eufemística de la relación gay en el periodismo serio", en Miguel Hernández Communication Journal, núm. 2, España: Universidad Miguel Hernández.

CIS (2010), Actitudes de la juventud ante la diversidad sexual. Disponible en: http://www. cis.es/cis/export/sites/default/Archivos/Marginales/2840_2859/2854/ Es2854.pdf [26 de octubre de 2018].

Clarke, Juanne N. (2006), "Homophobia out of the closet in the media portrayal of HIV/ AIDS 1991, 1996 and 2001: Celebrity, heterosexism and the silent victims", en Critical Public Health, vol. 16, núm. 4, Reino Unido: Taylor \& Francis.

Enguix, Begonya (2013), "Sexual Politics, Pride, and Media Mediation in Spain", en Observatorio $\left(O B S^{*}\right)$ Journal, vol. 7, núm. 2, Portugal: Universidad de Aveiro y OberCom. DOI: 10.15847/obsOBS722013651. Disponible en: http://dx.doi. org/10.15847/obsOBS722013651 [8 de enero de 2019].

Enguix, Begonya (2017), "Protesta, mercado e identidad en las celebraciones del Orgullo LGTB en España", en Convergencia. Revista de Ciencias Sociales, vol. 24, núm. 73, México: Universidad Autónoma del Estado de México. DOI: 10.29101/crcs. v0i73.4272. Disponible en: https://doi.org/10.29101/crcs.v0i73.4272 [10 de junio de 2018].

Etxazarra, Leire (2007), "La legalización del matrimonio homosexual (el cómo y el por qué de una movilización)”, en Papeles del CEIC, vol. 2007/1, núm. 26, España: Universidad del País Vasco. Disponible en: http://www.ehu.es/CEIC/pdf/26.pdf [29 de octubre de 2018].

Fairclough, Norman (2003), Analysing Discourse: Textual Analysis for Social Research, Reino Unido: Routledge.

Fairclough, Isabela y Fairclough, Norman (2012), Political Discourse Analysis: A Method for Advanced Students, Reino Unido: Routledge.

Fernández-Coronado, Ana (2006), "La evolución jurídica del sistema matrimonial español desde la Constitución de 1978 a la admisión del matrimonio homosexual", en Foro, Nueva Época, núm. 3, España: Universidad Complutense de Madrid.

Fundación BBVA (2007), Estudio Fundación BBVA sobre Actitudes Sociales de los Españoles. Disponible en: http://www.fbbva.es/TLFU/tlfu/esp/comunica/notas/fichanota/ index.jsp?codigo $=324$ [ 26 de octubre de 2018]. 
García-Pedraz, Javier (2012), "Siete años, 22.442 bodas y un recurso contra el matrimonio gay", en El Pais, 6 de noviembre de 2012. Disponible en: https://elpais.com/ sociedad/2012/07/13/actualidad/1342215460_536337.html [25 de octubre de 2018].

Giffney, Noreen y O'Rourke, Michael [eds.] (2017), The Ashgate Research Companion to Queer Theory, Reino Unido: Routledge.

Gouveia, Carlos Alberto (2005), "Assumptions about Gender, Power and Opportunity: Gays and Lesbians as Discursive Subjects in a Portuguese Newspaper", en Lazar, Michelle [ed.], Feminist Critical Discourse Analysis. Gender, Power and Ideology in Discourse, Inglaterra: Palgrave Macmillan.

Guijarro-Ojeda, Juan Ramón (2015), Análisis Crítico del Discurso en la prensa española: a propósito de la constitucionalidad del matrimonio entre personas del mismo sexo [tesis de Máster no publicada], España: Universidad de Granada.

Halliday, Michael y Matthiessen, Christian (2014), Halliday's introduction to Functional Grammar, Inglaterra y Estados Unidos: Routledge.

Jowett, Adam y Peel, Elizabeth (2010), "Seismic cultural change?: British media representations of same-sex 'marriage', en Women's Studies International Forum, núm. 33, Países Bajos: Elsevier.

Martín-Sánchez, María (2011), “Aproximación histórica al tratamiento jurídico y social dado a la homosexualidad en Europa”, en Estudios Constitucionales, vol. 9, núm. 1, Chile: Universidad de Talca.

Motschenbacher, Heiko y Stegu, Martin (2013), "Queer linguistic approaches to discourse", en Discourse and Society, núm. 24-5, Estados Unidos: Sage Journals.

Pan, Po-Lin et al. (2010), "Morality or equality? Ideological framing in news coverage of gay marriage legitimation”, en The Social Science Journal, vol. 3, núm. 47, Países Bajos: Elsevier.

Peterson, David (2011), "Neoliberal homophobic discourse: Heteronormative human capital and the exclusion of queer citizens", en Journal of Homosexuality, vol. 58, núm. 6-7, Reino Unido: Taylor \& Francis.

Richardson, John (2007), Analysing Newspapers. An Approach from Critical Discourse Analysis, Estados Unidos: Palgrave.

Sánchez-García, Francisco José (2012), El discurso referido en la prensa española, España: Octaedro.

Strand, Cecilia (2012), "Homophobia as a barrier to comprehensive media coverage of the Ugandan anti-homosexual Bill”, en Journal of Homosexuality, vol. 59, núm. 4, Reino Unido: Taylor \& Francis.

Tudela-Sancho, Antonio (2017), "Heteronormatividad y cuerpo sexuado: los placeres de la familia”, en Nuevo Itinerario. Revista Digital de Filosofía, vol. 7, núm. 7, Argentina: Universidad Nacional del Nordeste.

Van Dijk, Teun (2009), Discurso y poder, España: Gedisa. 


\section{Anexo}

\section{Tabla 1}

Total de procesos relacionados con personas LGTBI+ en corpus conservador

\begin{tabular}{lcccccc}
\hline \multicolumn{7}{c}{ Prensa conservadora } \\
\hline \multirow{2}{*}{ Proceso } & \multicolumn{2}{c}{ Positivo, neutro } & \multicolumn{2}{c}{ Negativo } & \multicolumn{2}{c}{ Total } \\
\cline { 2 - 8 } & $\mathrm{N}$ & $\%$ & $\mathrm{n}$ & $\%$ & $\mathrm{n}$ & $\%$ \\
\hline Material & 47 & 82,5 & 10 & 17,5 & 57 & 71,25 \\
\hline Mental & 5 & 62,5 & 3 & 37,5 & 8 & 10 \\
\hline Relacional & 4 & 50 & 4 & 50 & 8 & 10 \\
\hline Verbal & 7 & 100 & - & & 7 & 8,75 \\
\hline Conductual & - & & - & & - & \\
\hline Existencial & - & & - & & - & \\
\hline Total & 63 & 78,75 & 17 & 21,25 & 80 & 100 \\
\hline
\end{tabular}

Fuente: Elaboración propia.

\section{Tabla 2}

Total de procesos relacionados con personas LGTBI+ en corpus progresista

\begin{tabular}{lcccccc}
\multicolumn{7}{c}{ Prensa progresista } \\
\hline \multirow{2}{*}{ Procesos } & \multicolumn{2}{c}{ Positivo, neutro } & \multicolumn{2}{c}{ Negativo } & \multicolumn{2}{c}{ Total } \\
\cline { 2 - 8 } & $\mathrm{N}$ & $\%$ & $\mathrm{n}$ & $\%$ & $\mathrm{n}$ & $\%$ \\
\hline Material & 68 & 79,1 & 18 & 20,9 & 86 & 35,4 \\
\hline Verbal & 65 & 92,8 & 5 & 7,2 & 70 & 29 \\
\hline Mental & 44 & 74,6 & 15 & 25,4 & 59 & 24,4 \\
\hline Relacional & 13 & 68,4 & 6 & 31,6 & 19 & 7,8 \\
\hline Existencial & 2 & 40 & 3 & 60 & 5 & 2 \\
\hline Conductual & 3 & 100 & - & & 3 & 1,4 \\
\hline & 195 & 80,6 & 47 & 19,4 & 242 & 100
\end{tabular}

Fuente: Elaboración propia. 


\section{Gráfico 1}

Comparativa porcentual entre procesos del corpus conservador y progresista

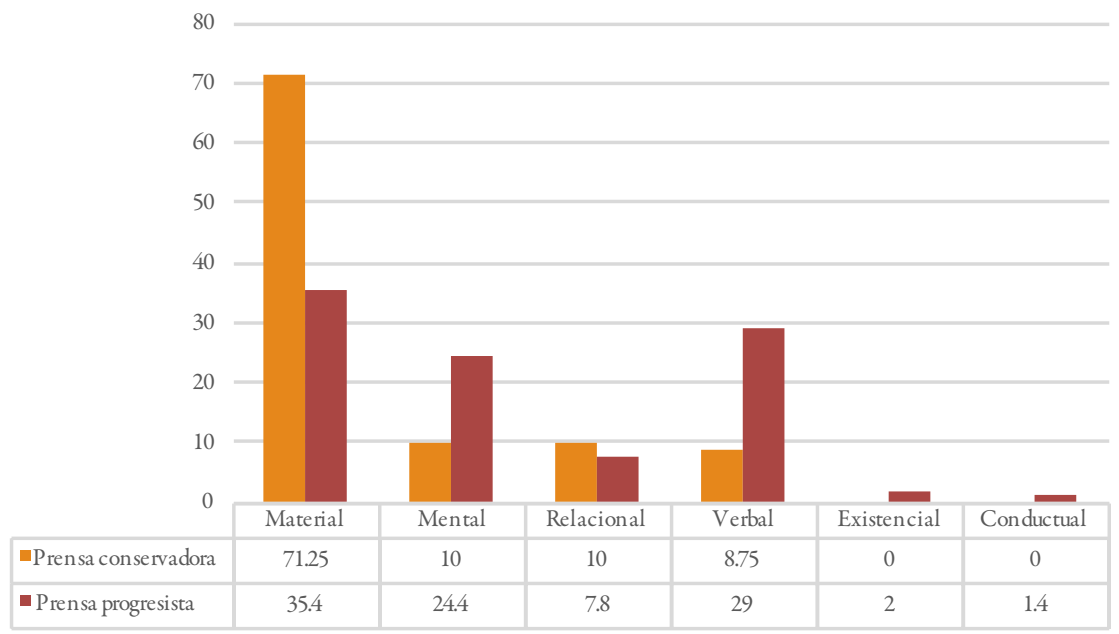

Fuente: Elaboración propia.

Juan Ramón Guijarro-Ojeda. Doctor en Didáctica de la Lengua y la Literatura; Profesor Titular del Departamento de Didáctica de la Lengua y la Literatura de la Universidad de Granada (España). Principales líneas de investigación: estudios de género en Lengua y Literatura, Análisis Crítico del Discurso, interculturalidad. Publicaciones recientes: Ruiz-Cecilia, Raúl y Guijarro-Ojeda, Juan Ramón (2016), “Lenguaje e ideología: a propósito de la nueva ley del aborto española”, en Convergencia. Revista de Ciencias Sociales, núm. 71, México: Universidad Autónoma del Estado de México; Barozzi, Stefano y Guijarro-Ojeda, Juan Ramón (2016), "Sexual identities in EFL at Primary School level: A pre-service teachers' perspective from Spain”, en Porta Linguarum, núm. 25, España: Grupo Editorial Universitario; Guijarro-Ojeda, Juan Ramón (2018), "Gender as a Global Issue in Foreign Language Teacher Training”, en Lopes, António y Ruiz-Cecilia, Raúl [eds.], New Trends in Foreign Language Teaching: Methods, Evaluation and Innovation, Reino Unido: Cambridge Scholars Publishing. 
Raúl Ruiz-Cecilia. Doctor en Didáctica de la Lengua y la Literatura. Profesor Titular y Director del Departamento de Didáctica de la Lengua y la Literatura de la Universidad de Granada (España). Principales líneas de investigación: Análisis Crítico del Discurso, lectura crítica en lengua extranjera, interculturalidad y género en educación. Publicaciones recientes: Ruiz-Cecilia, Raúl y Guijarro-Ojeda, Juan Ramón (2016), “Lenguaje e ideología: a propósito de la nueva ley del aborto española”, en Convergencia. Revista de Ciencias Sociales, núm. 71, México: Universidad Autónoma del Estado de México; Pérez-Valverde, Cristina y Ruiz- Cecilia, Raúl (2014), "Narrativas de la identidad docente en la formación del profesorado de lenguas extranjeras", en Andamios. Revista de Investigación Social, núm. 24, México: Universidad Autónoma de la Ciudad de México; Ruiz- Cecilia, Raúl (2011), "De la lectura intensiva a la extensiva en la clase de ILE. Fundamentos didácticos", en Odisea: Revista de Estudios Ingleses, núm. 12, España: Universidad de Almería. 